\title{
Development of a Low-Cost Medical Ultrasound Scanner Using a Monostatic Synthetic Aperture
}

\author{
Thomas L. A. van den Heuvel, David J. Graham, Kristopher J. Smith, \\ Chris L. de Korte, Senior Member, IEEE, and Jeffrey A. Neasham
}

\begin{abstract}
Objective: In this paper, we present the design of lowcost medical ultrasound scanners aimed at the detection of maternal mortality risk factors in developing countries. Method: Modern ultrasound scanners typically employ a high element count transducer array with multichannel transmit and receive electronics. To minimize hardware costs, we employ a single piezoelectric element, mechanically swept across the target scene, and a highly cost-engineered single channel acquisition circuit. Given this constraint, we compare the achievable image quality of a monostatic fixed focus scanner (MFFS) with a monostatic synthetic aperture scanner (MSAS) using postfocusing. Quantitative analysis of image quality was carried out using simulation and phantom experiments, which were used to compare a proof-of-concept MSAS prototype with an MFFS device currently available on the market. Finally, in vivo experiments were performed to validate the MSAS prototype in obstetric imaging. Results: Simulations show that the achievable lateral resolution of the MSAS approach is superior at all ranges compared to the fixed focus approach. Phantom experiments verify the improved resolution of the MSAS prototype but reveal a lower signal to noise ratio. In vivo experiments show promising results using the MSAS for clinical diagnostics in prenatal care. Conclusion: The proposed MSAS achieves superior resolution but lower SNR compared to an MFFS approach, principally due to lower acoustic energy emitted. Significance: The production costs of the proposed MSAS could be an order of magnitude lower than any other ultrasound system on the market today, bringing affordable obstetric imaging a step closer for developing countries.
\end{abstract}

Index Terms - Low cost imaging, obstetrics, single element, synthetic aperture, ultrasound.

\section{INTRODUCTION}

W ORLDWIDE, complications of pregnancy and childbirth lead to approximately 830 deaths every day, of

Manuscript received January 24, 2017; revised April 10, 2017; accepted April 11, 2017. Date of publication July 17, 2017; date of current version July 26, 2017. This work was supported in part by Delft Imaging Systems, in part by the Life Sciences \& Health for Development Fund, and in part by the UK Engineering and Physical Science Research Council. This paper was recommended by Associate Editor Dr. Mehdi Kiani. (Thomas L. A. van den Heuvel and David J. Graham contributed equally to this work.) (Corresponding author: Thomas L. A. van den Heuvel.)

T. L. A. van den Heuvel and C. L. de Korte are with the Medical UltraSound Imaging Centre (MUSIC), Department of Radiology and Nuclear Medicine, Radboud University Medical Centre, Nijmegen 6525 GA, the Netherlands (e-mail: Thomas.vandenHeuvel@ radboudumc.nl; Chris.deKorte@ radboudumc.nl).

D. J. Graham, K. J. Smith, and J. A. Neasham are with the School of Electrical and Electronic Engineering, Newcastle University, Newcastle upon Tyne NE1 7RU, United Kingdom (e-mail: dave.graham@newcastle.ac.uk; kristopher. smith@newcastle.ac.uk; jeff.neasham@newcastle.ac.uk).

Color versions of one or more of the figures in this paper are available online at http://ieeexplore.ieee.org.

Digital Object Identifier 10.1109/TBCAS.2017.2695240
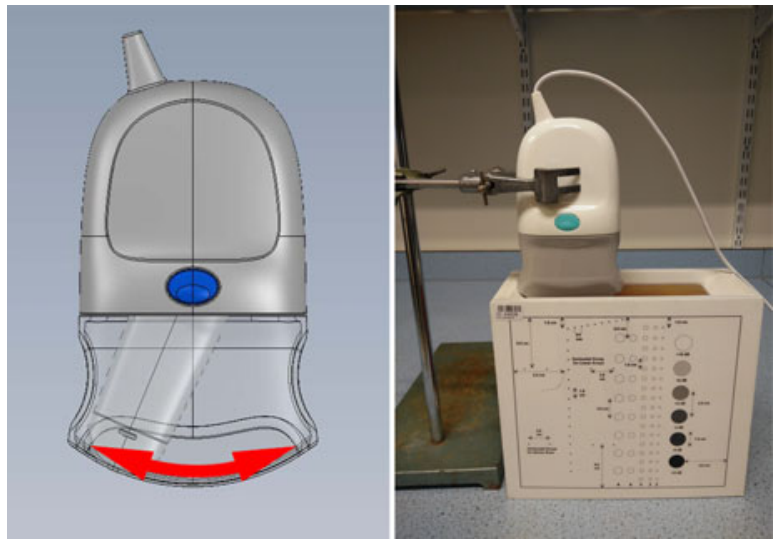

Fig. 1. Left: A schematic drawing of the MSAS design. The red arrow indicates the path of the single element transducer over the target scene. Right: experimental setup with the MSAS prototype for the phantom experiments.

which $99 \%$ occur in developing countries. This is mainly caused by the limited access to health services in these areas of the world [1]. With the use of ultrasound imaging it is possible to detect maternal mortality risk factors, but ultrasound devices remain out of reach for healthcare providers in low-resource settings because even the lowest cost ultrasound devices available on the market today are still cost prohibitive. Examples of low-cost devices recently entering the market include the Interson SeeMore probe, the SunBright SUN-806F and the Telemed MicrUs, which can be purchased between $\$ 1.5 \mathrm{k}$ and $\$ 3 \mathrm{k}$. The lowest cost devices emerging from the established ultrasound vendors include Siemens Acuson P10, GE Vscan, Philips Lumify and VISIQ, which are even more expensive options.

In this paper we present an ultrasound device with production costs less than $\$ 100$. This would make this ultrasound device an order of magnitude cheaper compared to the low-cost ultrasound devices available on the market today. To achieve this goal, a significant reduction in complexity of the hardware is required. The main cost driver of ultrasound systems is the multi-element piezoelectric transducer array which is intricate, expensive and also requires multiple channels of multiplexed transmit and receive electronics. Hardware costs can be vastly reduced by simplifying the transducer array to a single piezoelectric element, as shown in Fig. 1, which is mechanically swept across the target scene. 


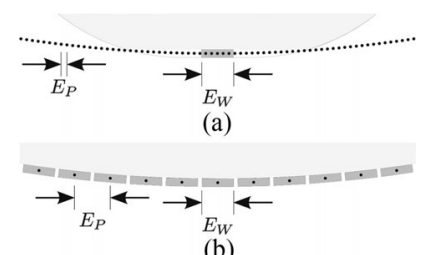

(b)

Fig. 2. Comparison of array geometry using a physical element of width, $E_{W}$. Each element position is indicated by a dot, resulting in the effective element pitch distance, $E_{P}$. (a) Convex synthetic array formed from a single moving element. (b) Convex physical array.

A monostatic design, consisting of a single, mechanically scanned transmit/receive transducer element, poses two main challenges. Firstly, the maximum achievable frame rate will be limited compared to electronic beam-steering, because the single element has to be moved across the target scene. However, as long as this frame rate is sufficient for the diagnostic task in hand, the reduction in production costs outweighs this disadvantage. Second, a monostatic design leads to compromises on lateral resolution compared to an electronically focused transducer array, with resolution constrained by the fixed beam pattern of a naturally focused transducer and the sub-optimal resolution in the near and far field regions. To improve the lateral resolution of the monostatic design, synthetic aperture focusing is explored here.

Besides the lower production costs, the monostatic design may offer some advantages compared to a full array transducer. The response of a monostatic system is perfectly matched at each position and should also be more reliable as individual elements of a transducer array can fail over time and degrade image quality [2]. Given the intended low resource setting, the likelihood of probe damage is increased, hence a cheap and easily replaceable transducer arrangement is preferable. A monostatic system may also allow a synthetic array with an element pitch, $E_{P}$, smaller than the physical element width, $E_{W}$, which is not possible with a physical array, as shown in Fig. 2.

The monostatic design was evaluated using simulations, phantom experiments and in vivo experiments. Simulations were made to compare the fundamental performance of MFFS and MSAS designs. A proof-of-concept MSAS was then produced and compared a MFFS ultrasound device via phantom experiments. In vivo experiments were performed to validate the use of the MSAS prototype in prenatal care.

\section{LITERATURE OVERVIEW}

Most reported work on synthetic aperture focusing in ultrasound imaging uses a physical transducer array [3]-[7]. The use of synthetic aperture focusing in medical imaging was introduced by Burckardt et al. [8] who showed that synthetic aperture with a single element gives a significantly higher lateral resolution compared to a conventional B scan. In 2007, Kortbek et al. [9] used Field II simulations to show that a single rotating mechanically focused concave element, which is used in an anorectal ultrasound transducer, increased the SNR. In 2010 Opretzka et al. [10] used a fixed focus single-element for high frequency ultrasound on a wire phantom and showed

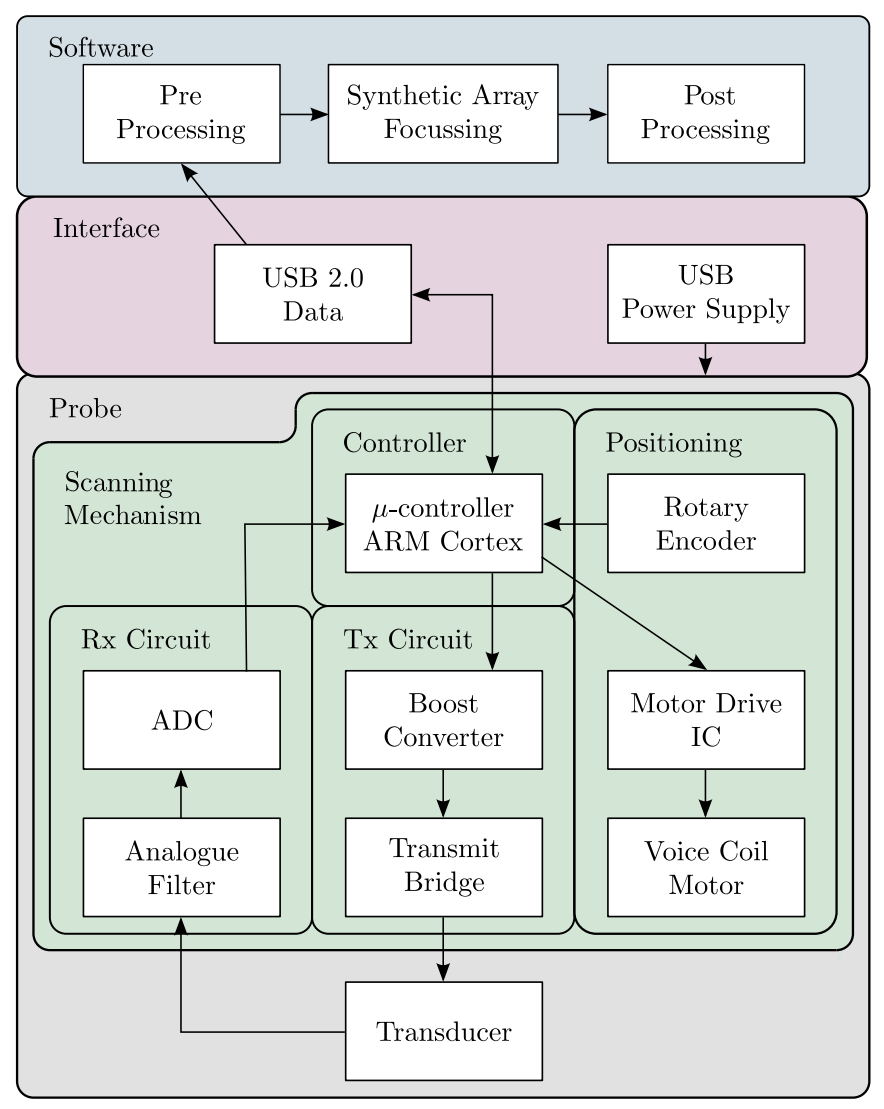

Fig. 3. A schematic overview of the MSAS prototype design.

a significant reduction of side lobes and of noise compared to delay-and-sum. (They have also a paper in 2012 that shows animal results [11]). In 2011 Andresen et al. [12] used synthetic aperture focusing with a single-element transrectal ultrasound transducer, making a helical motion to obtain 3-D volumes. Simulations and a wire phantom experiment showed a significant improvement in azimuth resolution. Although these papers describe the use of synthetic aperture focusing for medical imaging with a single element, none of this work has shown any In vivo results of this technique and this is the first low-cost device using synthetic aperture focusing [13] applied on prenatal care in developing countries.

\section{METHODS}

The MSAS prototype and testing methodology is presented in three sections: hardware design, software design and simulation/experiments. A schematic overview of the hardware and software is shown in Fig. 3.

The hardware section describes the design of the low cost probe including the transducer, the scanning mechanism and the communication interface. The software section describes the processing of the raw data, acquired by the low cost probe, to produce the B-mode image, which includes the synthetic aperture focusing method. The simulations and experiments section describes simulations, phantom and in vivo experiments that were performed to evaluate the image quality of the MSAS. 
TABLE I

MSAS PROTOTYPE PARAMETERS

\begin{tabular}{lcc}
\hline \hline Name & Symbol & Value \\
\hline Centre Frequency & $f_{c}$ & $4.2 \mathrm{MHz}$ \\
Bandwidth & $\Delta f$ & $2 \mathrm{MHz}$ \\
Range & $R$ & $0.15 \mathrm{~m}$ \\
Average speed of sound in tissue & $c$ & $1540 \mathrm{~ms}^{-1}$ \\
\hline Radius of Curvature & & $0.082 \mathrm{~m}$ \\
Lateral Arc Length & $\theta_{L}$ & $50^{\circ}$ \\
Angular Resolution & $\theta_{\mathrm{res}}$ & $0.25^{\circ}$ \\
\hline ADC Resolution & $\mathrm{ADC}$ res & $14 \mathrm{bit}$ \\
Sampling Frequency & $f_{s}$ & $12 \mathrm{MS} / \mathrm{s}$ \\
\hline Element Width & $E_{W}$ & $2 \mathrm{~mm}$ \\
Element Length & $E_{L}$ & $7 \mathrm{~mm}$ \\
Element Thickness & $E_{T}$ & $0.5 \mathrm{~mm}$ \\
Near Field Lateral & $N_{L}$ & $2.7 \times 10^{-3} \mathrm{~m}$ \\
Near Field Elevation & $N_{E}$ & $0.034 \mathrm{~m}$ \\
Beam Spread Angle Lateral & $\alpha_{L}$ & $10.8^{\circ}$ \\
Beam Spread Angle Elevation & $\alpha_{E}$ & $3.1^{\circ}$ \\
\hline Frames/s (software limited) & & 4 \\
Data Throughtput (4FPS) & & $30 \mathrm{Mb} / \mathrm{s}$ \\
\hline \hline
\end{tabular}

\section{A. Probe Hardware}

1) Transducer: The first major step toward lower cost hardware is to replace the costly construction of a multi-element piezoelectric transducer array and multiple channels of transmit and receive electronics with a single element transducer and single channel of electronics, similar to early imaging systems [8], [14].

The monostatic design significantly simplifies the transducer construction to a single piece of piezoelectric material with an electrical connection to each electrode, a quarter-wave matching layer on the active face and an attenuative backing material on the opposite face. The axial resolution is determined by the bandwidth, $\Delta f$, whereas the lateral and elevation beam width are determined by the size and geometry of the transducer aperture and the centre frequency, $f_{c}$. The physical size of the transducer chosen for the MSAS design is strongly related to the synthetic aperture focusing process which will be explained in Section III-C2. Operating frequencies of $2-5 \mathrm{MHz}$ are typical for ultrasound abdominal probes as this represents the best tradeoff between resolution and penetration depth. In this design the transducer makes direct contact with the skin. With careful design of the transducer housing shape, it is found that coupling to the skin can be maintained via standard coupling gel.

2) Scanning Mechanism: The single element must be mechanically moved along a predetermined path in order to collect the necessary echo data to build an image. This path was chosen to match that of a standard convex array probe, as shown in Fig. 1, which represents the best trade-off between the probe dimensions and the field of view. The transducer of the MSAS prototype makes a sweep of $50^{\circ}$ on an $8 \mathrm{~cm}$ radius arc corresponding to a 200 element synthetic array. It takes $0.25 \mathrm{~s}$ to obtain one sweep, which results in a frame rate of four frames per second (see Table I for an overview of all parameters).

a) Positioning system: A motor is used to sweep the single transducer across the target scene. The motor design has four design criteria: high torque to overcome the contact friction with the skin, low electrical noise so it does not adversely affect the Signal-to-Noise Ratio (SNR) of echo data, a maximum current of $300 \mathrm{~mA}$ for operation from a single USB bus power supply and finally appropriate size/weight for the probe to be easily held with one hand. A Voice Coil Motor (VCM) was designed to satisfy these requirements [15]. This motor design uses no brushes and hence is electrically quiet. Furthermore, direct drive means there is no audible noise from a gearbox and there are minimal parts which will suffer mechanical wear. The torque generated by the motor is limited, as described in [15], and if too much pressure is applied by the operator the motor stalls before any discomfort or injury could result. Fluctuations in speed due to variable friction have no inherent effect on image quality as the optical encoder determines when the transducer is excited to ensure that the data is accurately captured.

Accurate position registration was achieved using an optical rotation encoder with an angular resolution, $\theta_{\text {res }}$, which then initiates each transmit- and receive cycle at $0.25^{\circ}$ increments.

Basic closed loop speed control was implemented, using a pulse-width modulation motor driver together with feedback from the rotary encoder, in order to achieve near uniform sampling of the synthetic array.

b) Transmit Electronics: The transmit circuit is required to produce a short high voltage pulse to excite the transducer at its resonance. The duration of the pulse in cycles should be the upper bound of the $\mathrm{Q}$ factor of the transducer (1).

$$
Q=\frac{f_{c}}{\Delta f}
$$

A boost converter was used to produce $48 \mathrm{~V}$ from the $5 \mathrm{~V}$ USB supply which was then used to supply a class-E amplifier. The inductor in this amplifier topology was selected to provide a Q-magnification of 2, hence producing a $96 \mathrm{~V}$ 2-cycle pulse at $4.2 \mathrm{MHz}$ to drive the transducer.

c) Receive Electronics: The data acquisition sub-system is the most expensive electronic component of a medical ultrasound device, typically requiring multiple channels of high bandwidth, low noise amplifiers/filters and high specification Analogue to Digital Converters (ADCs). Even when simplifying to a single channel, these components still represent a large proportion of the overall system cost. Careful design and performance tradeoffs must be considered to achieve a truly low cost device.

The ADC of the MSAS design was chosen as the optimum balance between price and performance in terms of signal to noise ratio as estimated by the ideal ADC equation (2), where $b$ is the bit resolution. Based on these criteria 14-bit ADC was selected capable of sampling at $12 \mathrm{MS} / \mathrm{s}$ which costs $<\$ 10$ in large quantities.

$$
\mathrm{ADC}_{\mathrm{SNR}}=1.76+6.02 b+10 \log _{10}\left(\frac{f_{s}}{2 \cdot \Delta f}\right) \mathrm{dB}
$$

d) Controller: The controller is synchronises all positioning, ultrasound transmission and data acquisition operations and transfers echo and position data to the processing software. 


\section{B. Interface}

The MSAS probe was designed to interface to a standard USB 2.0 connection which provides sufficient data rate and power supply for this design. This ensures compatibility with many processing platforms, whether new or legacy.

\section{Software}

The processing software was developed to perform the signal processing and display the B-mode image on readily available, low-cost platforms such as PCs and laptops. Given the wide availability of PCs and the number of schemes already in place to provide hardware to the developing world, it is believed that this represents the cheapest possible processing and display unit, which lowers the overall system costs. First, the echo data is filtered and down-converted. Second, synthetic aperture focusing is applied to the baseband data. Last, post-processing steps are performed to generate the final grey scale, B-mode image.

1) Pre-Processing: After formatting the echo data, band pass filtering is applied over a bandwidth of $2 \mathrm{MHz}$ around the centre frequency, $f_{c}$, to remove out of band energy from the data. Then a time varying gain curve is applied to the echo data to compensate for attenuation with depth. Finally the echo data is down-converted to a complex baseband representation.

2) Synthetic Aperture Focusing: When using a fixed focus approach with a single mechanically swept transducer, the Bmode image is constructed by a simple polar to Cartesian conversion of echo data from each direction viewed. The lateral resolution of such a system is dependent on the beam spread angle, $\alpha$ which favours a large aperture diameter, $E_{D}$ (3). However, the image will also be distorted due to the complex beam shape up to the near field distance, $D_{\text {near }}$, given by (4) which is proportional to the square of $E_{D}$. Hence any fixed focus system represents a trade-off between the extent of the near field and the beam spread in the far field which suggests that the optimum fixed focus transducer for typical obstetric imaging, at $4 \mathrm{MHz}$ and up to $15 \mathrm{~cm}$ range, consists of $\mathrm{a} \approx 15 \mathrm{~mm}$ diameter ceramic disk with an acoustic lens to bring the natural focus back to $\approx 7 \mathrm{~cm}$.

$$
\begin{aligned}
\frac{\alpha}{2} & =\sin ^{-1}\left(\frac{0.514 \cdot c}{f_{c} \cdot E_{D}}\right) \\
D_{\text {near }} & =\frac{E_{D}^{2} \cdot f_{c}}{4 \cdot c}
\end{aligned}
$$

The MSAS uses synthetic aperture focusing whereby echo data from multiple positions are coherently summed to calculate each image pixel. This favours a transducer with a larger beam spread angle in the lateral direction i.e. the direction of motion, but still requires a narrow beam in the elevation plane. With the use of synthetic aperture focusing each individual pixel $_{i j}$ of an image of size $i \times j$ can be calculated as shown in Fig. 4 by combining the received baseband echo data, $B_{i j}$ from $N$ synthetic aperture positions from which that pixel is visible and applying appropriate delays, $d_{n}$, phase rotations, $\phi$, and weightings, $W_{i j}$, as given by (5). The sample delay, $d_{n}$, is calculated as in (6) with the two-way path length, $L_{p}$, the sampling frequency, $f_{s}$, and the average speed of sound in tissue $c$. The phase rotation,

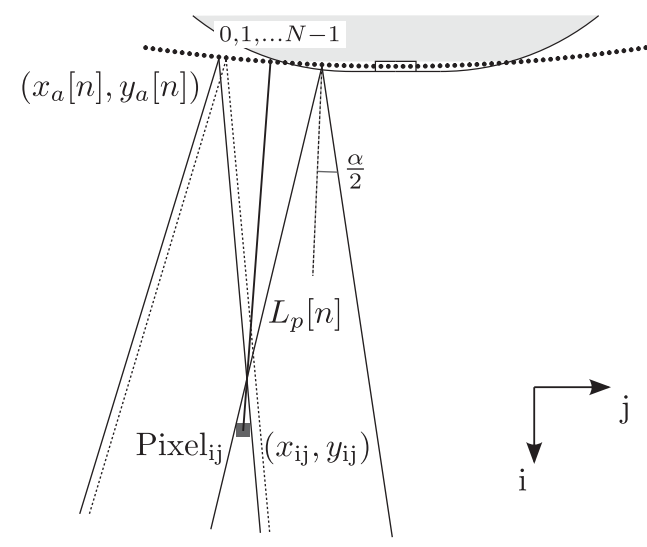

Fig. 4. Synthetic aperture focusing to calculate Pixel $_{i j}$ from $\mathrm{N}$ synthetic aperture elements.

$\phi$, is calculated as in (7) using the centre frequency, $f_{c}$. The two way path length, $L_{p}$, is calculated using (8) where $\left(x_{a}, y_{a}\right)$ and $\left(x_{f}, y_{f}\right)$ are the $x$ and $y$ coordinates of the aperture element and the coordinates of focus respectively. The weightings, $W_{i j}$, are selected from an $N$ length Tukey window function [16] to achieve an acceptable trade-off between main lobe width and side lobe levels.

$$
\begin{aligned}
\operatorname{Pixel}_{i j} & =\left|\frac{1}{N} \cdot \sum_{n=0}^{N-1} W_{i j}[n] \cdot B_{i j}\left[n, d_{n}\right] \cdot \phi_{i j}[n]\right| \\
d_{n} & =\left|\frac{L_{p}[n] \cdot f_{s}}{c}\right| \\
\phi_{i j}[n] & =\frac{-2 \cdot \pi \cdot L_{p}[n] \cdot f_{c}}{c} \\
L_{p}[n] & =2 \cdot \sqrt{\left(x_{i j}^{2}-x_{a}^{2}[n]\right)^{2}+\left(y_{i j}^{2}-y_{a}^{2}[n]\right)^{2}}
\end{aligned}
$$

There are two key advantages of this technique. Firstly, the effective transducer aperture increases proportional to the axial range which makes lateral resolution less dependent on range. In the case of a linear scan path, the lateral resolution becomes independent of range but in the case of a convex array some degradation of lateral resolution with respect to range remains, depending on the radius of curvature. Secondly, synthetic focusing of signals gathered from a small physical aperture ensures that the best possible beam pattern is formed at every range and eliminates the distortions seen in the near field of a larger physical aperture.

3) Post-Processing: Two straightforward post-processing steps were performed to improve the visual quality of the Bmode image for clinical use. First, a log compression was performed to adjust the dynamic range. Second, a stick filter [17] was applied to reduce coherent speckle in the final image.

\section{Simulations and Experiments}

Simulations and experiments were performed to evaluate the MSAS design. First, Field II simulations were used to compare the image quality of MSAS versus MFFS. Second, phantom experiments were used for quantitative comparison of the MSAS 
prototype with an MFFS ultrasound device (Interson SeeMore, model 99-5901, centre frequency 3.5 MHz). The MFFS ultrasound device makes use of a fixed focus single element which moves inside an oil filled housing. Finally, in vivo experiments were used to validate the use of the MSAS prototype for common measurements in prenatal care.

1) Simulations: Field II [18], [19] was used to simulate a single element ultrasonic transducer travelling a convex path with an angular resolution, $\theta_{\text {res }}$, of $0.25^{\circ}$ and performing a transmitand receive cycle at each position. For MSAS a rectangular element of $2 \mathrm{~mm}$ (lateral) by $7 \mathrm{~mm}$ (elevation) was simulated whereas for the MFFS simulation a circular aperture of $15 \mathrm{~mm}$ diameter was used together with a lens, giving similar beam characteristics to the MFFS ultrasound device.

2) Phantom Experiments: The image quality of the MSAS prototype and MFFS ultrasound device were evaluated using Quality Assurance for UltraSound (QA4US) software. This software makes it possible to quantitatively analyse B-mode images and was used to measure the elevation focus, spatial resolution, spatial conformity and contrast sensitivity of each ultrasound device [20].

a) Elevation focus: The elevation direction of the ultrasound probe is perpendicular to the displayed sector and therefore normally not visible. However, special slice thickness phantoms have been developed to visualize the elevation focus using a plane of scatterers which are positioned at an angle of $45^{\circ}$. This enables the visualization of the slice thickness as a function of the depth of the scatterers in the phantom. The QA4US software defines the elevation focus depth as the depth where the smallest detected slice thickness is located.

b) Spatial resolution: The QA4US software defines the spatial resolution of an ultrasound device as the Full Width Half Maximum (FWHM) of the wire in the phantom that is closest to the elevation focus. The depth of the (in-plane) transmit focus was also set to the elevation focus depth. This will result in the best spatial resolution that can be imaged with the device. The spatial resolution degredates outside the focal point. This degradation was quantified by taking the average of the FWHM of the wires in the phantom two centimetres above and below the focal point and compare this to the best spatial resolution that can be imaged with the device.

c) Spatial conformity: The QA4US software defines the spatial conformity as the percentage difference between two measured wires in the phantom measured on the B-mode image and the actual distance of the wires in the phantom.

d) Dynamic range and contrast sensitivity: The QA4US software defines the dynamic range as the number of $\mathrm{dB}$ 's within the 0-255 gray level range. It was measured by acquiring multiple images of the contrast objects in a phantom. The contrast sensitivity is defined as the SNR at $3 \mathrm{~dB}$ as shown in (9)

$$
\mathrm{SNR}_{\mathrm{L}}=\frac{\left|\mu_{L}-\mu_{B}\right|}{\sqrt{\sigma_{\mu_{\mathrm{L}}}^{2}+\sigma_{\mu_{\mathrm{B}}}^{2}}}
$$

where: $\mu_{L}$ and $\mu_{B}$ are the ensemble mean echo levels of lesion (L) and the surrounding background tissue (B) [20]. This makes the contrast sensitivity dependent on the dynamic range.
Therefore the dynamic range needs to be corrected to one reference dynamic range to be able to make a fair comparison of the contrast sensitivity between different ultrasound devices. All pixels in the image are multiplied according to (10) to produce a corrected pixel value $\operatorname{Pixel}_{i j}^{\prime}$, where $\operatorname{Pixel}_{i j}$ is the original pixel intensity of a pixel, $\mathrm{DR}_{\mathrm{ref}}$ is the reference dynamic range of 2.55 and DR is the measured dynamic range of the device.

$$
\operatorname{Pixel}_{i j}^{\prime}=\operatorname{Pixel}_{i j} \cdot \frac{\mathrm{DR}_{\mathrm{ref}}}{\mathrm{DR}}
$$

3) In Vivo Experiments: In vivo experiments were performed to evaluate the performance of the MSAS prototype in prenatal care. The local ethics committee approved the use of the MSAS prototype on pregnant women. This was achieved by proving that the SESAS provides conformance to the FDA Track 1 standards Fetal Imaging application. Hydrophone measurements showed a Mechanical Index below 0.2, a Derated Spatial-Peak Temporal-Average Intensity below $9 \mathrm{mWcm}^{-1}$ and a Derated Spatial-Peak Pulse-Average Intensity below $5.2 \mathrm{Wcm}^{-1}$. The electrical safety of the system was tested according to the NENEN-IEC 60601-1 of the NEN 3140. Every pregnant woman in this evaluation study signed a written informed consent. In prenatal care ultrasound can be used to detect maternal mortality risk factors. The performed in vivo experiments focussed on biometric measurements that can be used to determine the Gestational Age (GA) and growth of the fetus. In the first trimester the Crown-Rump Length (CRL) of the fetus is the most reliable measurement to determine the GA. The Head Circumference (HC) and Abdomen Circumference (AC) of the fetus can be used in the second and third trimester to assess the growth of the fetus [21].

\section{RESUlTS}

\section{A. Simulation}

Fig. 5 shows the results of Field II simulations to generate B-mode images of an arrangement of ideal point targets, similar to that used in phantom experiments. The left image shows the MSAS result and the right image shows the MFFS result. Fig. 6 shows a comparison of the lateral resolution versus depth computed from the vertical row of the images in Fig. 5.

\section{B. Phantom Experiments}

Fig. 7 shows an example of point target data, at a depth of $10 \mathrm{~cm}$, extracted from the resolution phantom experiments with the MSAS prototype. The improvements in lateral resolution and signal to noise ratio resulting from synthetic focusing are clearly shown.

Figs. 8 and 9 show B-mode images obtained in phantom experiments using the MSAS prototype and the MFFS ultrasound device. Quantitative analysis of the image quality from the two ultrasound devices was performed using the QA4US tool. Table II shows the result of this analysis. 


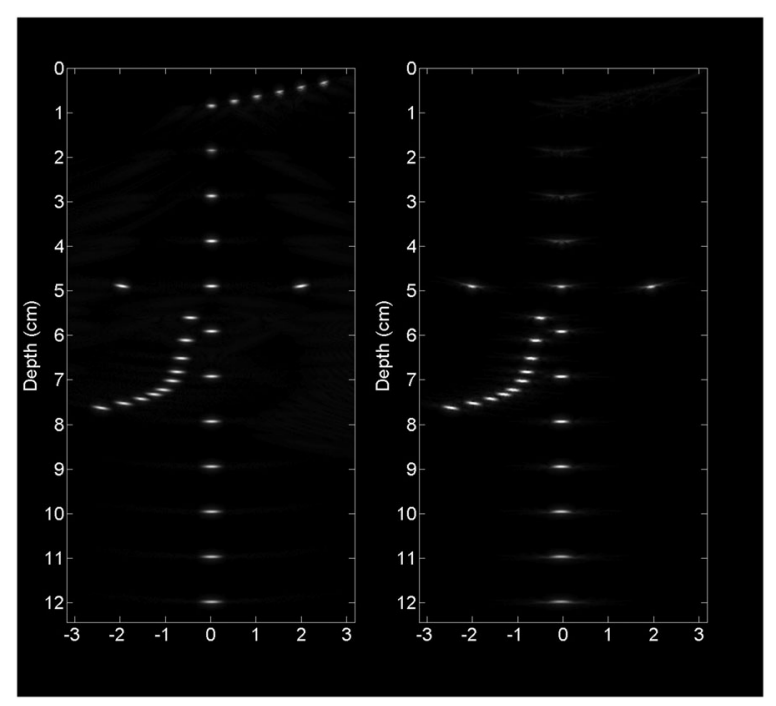

Fig. 5. Simulated images of point targets. Left: MSAS. Right: MFFS.

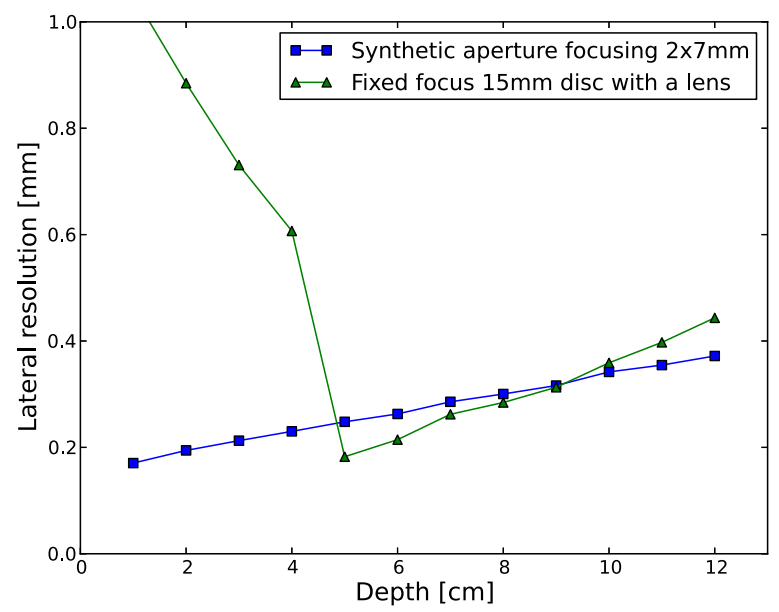

Fig. 6. Lateral resolution computed from the simulated images of point targets.

\section{In Vivo Experiments}

Fig. 10, shows three example B-mode images of the in vivo experiments with the MSAS prototype, one from each of the trimesters of the pregnancy. The top image shows a side view of a fetus in the first trimester, which can be used to measure the crown to rump length (CRL) of a fetus. The middle image shows a cross section of the fetal head in the second trimester, which can be used to measure the head circumference $(\mathrm{HC})$ of the fetus. The bottom image shows a cross section of the fetal abdomen in the third trimester, which can be used to measure the abdominal circumference (AC) of the fetus.

\section{DISCUSSION}

\section{A. Simulations}

The Field II simulations, shown in Figs. 5 and 6, show that the best case lateral resolution of the MSAS and MFFS approaches is similar. However it is clearly demonstrated that the near field distortions seen on the MFFS image, due to the complex beam
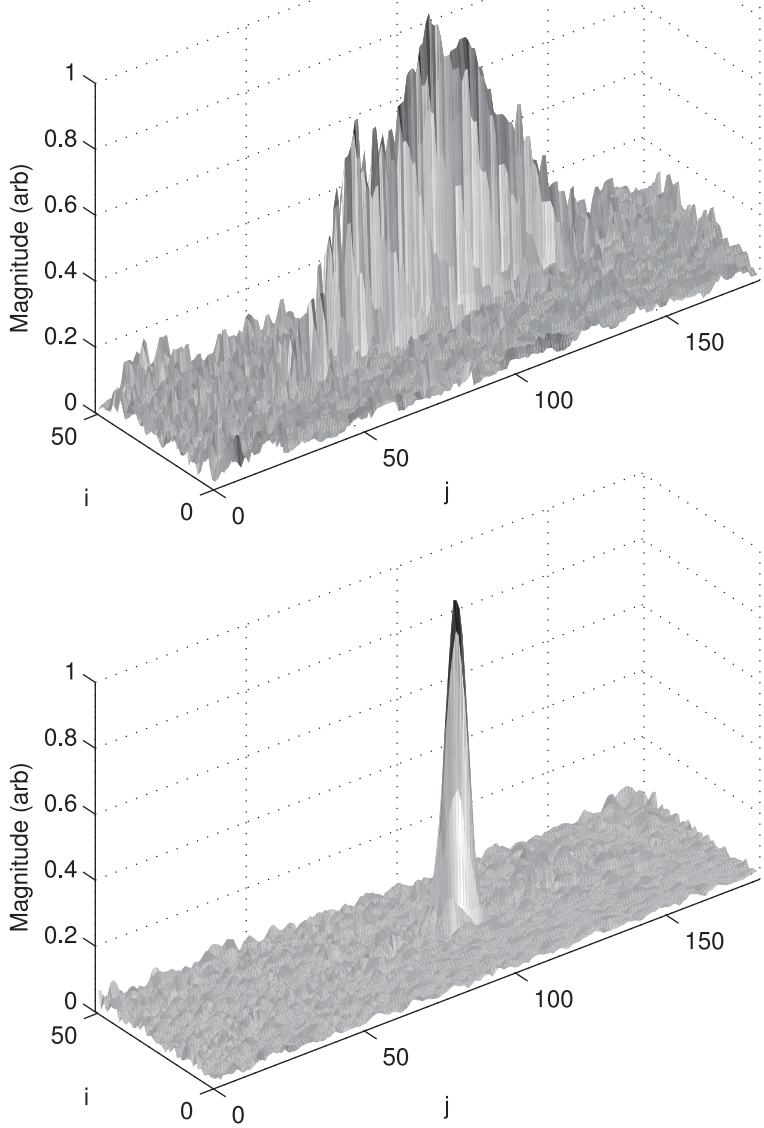

Fig. 7. Surface plot of a $0.1 \mathrm{~mm}$ diameter target from a resolution phantom at a depth of $10 \mathrm{~cm}$ obtained using MSAS prototype. (a) Physical beam pattern (before synthetic focusing). (b) After synthetic focusing.

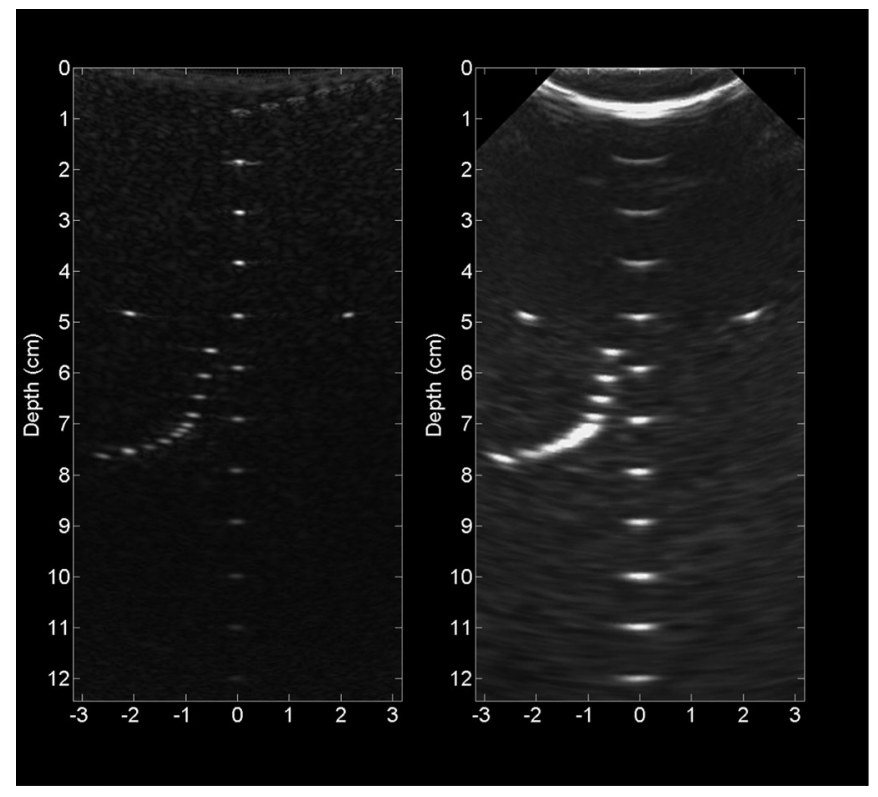

Fig. 8. Example images of the phantom wires used for calculating spatial resolution and spatial conformity. Left: MSAS prototype. Right: MFFS ultrasound device. 


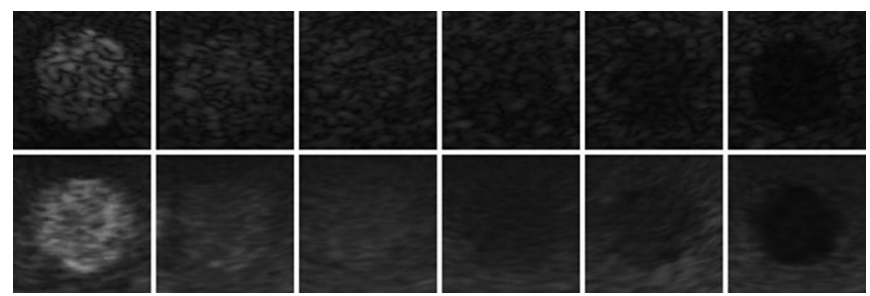

Fig. 9. Example images of the phantom contrast disks that are used for calculating the dynamic range and contrast sensitivity (left to right: $15 \mathrm{~dB}$, $6 \mathrm{~dB}, 3 \mathrm{~dB},-3 \mathrm{~dB},-6 \mathrm{~dB},-15 \mathrm{~dB})$. Top: MSAS prototype. Bottom: MFFS ultrasound device.

TABLE II

QA4US RESULTS

\begin{tabular}{lcc}
\hline \hline Parameter & MSAS & MFFS \\
\hline Elevation Focus [mm] & 30.6 & 50.5 \\
Slice thickness at elevation focus [mm] & 1.08 & 1.2 \\
\hline Axial Resolution in focus [mm] & $0.23 \pm 0.03$ & $0.37 \pm 0.05$ \\
Axial Resolution averaged over depth [mm] & $0.28 \pm 0.05$ & $0.55 \pm 0.13$ \\
\hline Lateral Resolution in focus [mm] & $0.55 \pm 0.02$ & $1.25 \pm 0.06$ \\
Lateral Resolution averaged over depth [mm] & $0.67 \pm 0.11$ & $2.71 \pm 1.40$ \\
\hline Axial spatial conformity [\%] & $0.2 \pm 0.18$ & $2.0 \pm 0.06$ \\
\hline Dynamic Range [dB] & 140 & 98 \\
Contrast Sensitivity & 1.22 & 2.20 \\
Corrected Contrast Sensitivity & 1.46 & 2.19 \\
\hline \hline
\end{tabular}

shape up to the near field distance, are eliminated using the MSAS approach and the resolution at the maximum range is also improved. Overall, the MSAS approach achieves a much more consistent lateral resolution over the full range of the image.

\section{B. Phantom Experiments}

The MSAS prototype demonstrates superior axial and lateral resolution compared to the MFFS ultrasound device, which is visible in Fig. 8 and was quantified with the QA4US software with results shown in Table II. This superior resolution becomes even more pronounced when the mean resolution over the full depth range is evaluated. The MFFS ultrasound device has a large decay in lateral resolution due to its fixed focus, single element transducer design. The mean lateral resolution of the MFFS ultrasound device is therefore more than twice as poor as the best case resolution.

The spatial conformity in axial direction of the MFFS ultrasound device was also found to be worse compared to the MSAS prototype. This may be simply due to the poorer lateral resolution but it may also be affected by the applied frame averaging or smoothing. Unfortunately, post-processing could not be turned off by the software.

After correction for dynamic range it can be seen that the contrast sensitivity of the MSAS prototype is significantly lower compared to the MFFS ultrasound device. This is caused by the combination of the small aperture size of the transducer element $(2 \mathrm{~mm} \times 7 \mathrm{~mm})$ and compromises in the analog front
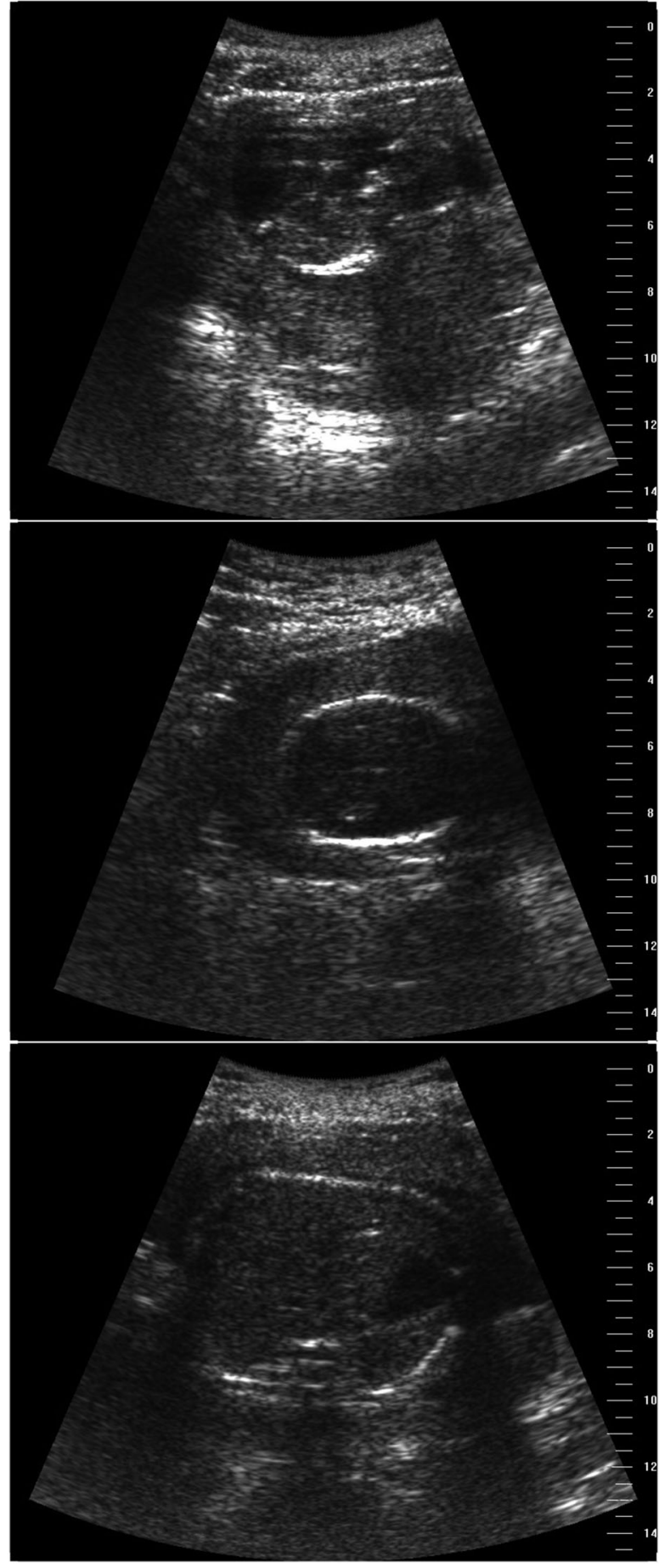

Fig. 10. From top to bottom: B-mode image of a fetus in the first trimester, which can be used to measure the CRL of the fetus. B-mode image of the head of a fetus in the second trimester, which can be used to measure the HC. B-mode image of the abdomen of a fetus in the third trimester, which can be used to measure the AC.

end electronics, both of which degrade the achievable SNR even after focusing.

\section{In Vivo Experiments}

Extensive in vivo experiments have shown that it is possible to view the fetus with the MSAS prototype in all trimesters, 
illustrated by the three examples in Fig. 10. The relatively low contrast sensitivity of the MSAS makes it a more challenging task to determine the exact border of the fetus in the first trimester and therefore error bounds on CRL measurements are likely to be higher than with state-of-the-art scanners. The middle image of shows that the MSAS is able to image a sharp edge of the fetal head but limited detail of the internal soft tissue structure, again due to the contrast sensitivity. This makes it more challenging to find internal markers which indicate the ideal cross section of the fetal head for the $\mathrm{HC}$ measurement but a reasonable estimate of the head circumference can still be made. In the third trimester it proved possible to view the abdomen of the fetus, but it is more challenging to pick out all deeper structures which are not as clearly visible due to shadowing and the lower contrast sensitivity. Despite the lower frame rate and the lower contrast of the MSAS, it has still proven possible to measure important fetal biometrics such as CRL, HC and $\mathrm{AC}$.

\section{User Experience}

When designing a low cost ultrasound devices compromises have to be made to decrease production costs. Like most low cost ultrasound devices, the MSAS prototype has relatively few parameters that can be adjusted by the user. This may make the use of these devices in developing countries easier but it is not possible to change them if parameters are suboptimal. A significant compromise of the MSAS prototype constructed was found to be the lower frame rate. This diminishes the 'realtime' imaging experience that experienced sonographers are accustomed to. Users could adapt to this lower frame rate but, in combination with the low contrast sensitivity, this made it more difficult and time consuming to locate the appropriate views of a fetus for biometric measurements.

\section{E. Improvements}

The biggest scope for improvement on the MSAS design is in the contrast sensitivity of the device by increasing the received SNR. Three main approaches could be used to obtain a higher contrast sensitivity. Firstly, a larger transducer aperture in the elevation direction in combination with a lens to optimise the focal depth, would increase the transmitted and received ultrasound energy. Second, a higher specification ADC could be used to allow oversampling well above the Nyquist sampling frequency to reduce aliasing and quantisation noise. Thirdly, there is substantial scope to improve to the receiver amplifier to reduce noise floor. User experience would also be improved by a higher frame rate which is possible by optimisation of the processing software. However, careful design will be needed, harnessing ever improving cost/performance of components, to maintain an order of magnitude reduction of system cost. At the time of writing only simple image post-processing steps are performed in the MSAS software. More advanced post-processing steps could be also included to improve the visual quality of the images by optimising dynamic range and reducing speckle.

\section{CONCLUSION}

In this paper a low component cost, monostatic synthetic aperture scanner (MSAS) was presented with potential applications in low resource countries for the detection of maternal risk factors. This system was designed to reduce the production costs by replacing the multi-element piezoelectric transducer array by a single piezoelectric element which is mechanically swept across the target scene. Since a single piezoelectric element leads to inevitable compromises in image resolution, synthetic aperture focusing was used to achieve consistently high resolution across the B-mode image. Simulations proved that the lateral resolution of the monostatic synthetic aperture focusing approach is superior to that of a monostatic fixed focus scanning (MFFS) approach at almost every range. Phantom experiments, performed with a proof-of-concept MSAS prototype, showed that it has superior axial and lateral resolution compared to another single element 'low cost' ultrasound device that is available on the market today. However, the phantom experiments also showed that the current MSAS design suffered from a relatively low contrast sensitivity. To validate the performance of the MSAS in prenatal care in vivo experiments were performed. The in vivo experiments show promising results for clinical diagnostic use of the MSAS. Even with the lower frame rate is was possible to detect the fetus in all three trimesters and image different parts of the fetus that are important for making biometric measurements of the fetus. Therefore it can be concluded that, with further development, the proposed design has the potential to deliver an affordable technology for developing countries to detect maternal risk factors and hopefully reduce the number of maternal deaths in the future.

\section{REFERENCES}

[1] “Maternal mortality,” World Health Organization, fact sheet No.348, 2015. [Online]. Available: http://www.who.int/mediacentre/factsheets/fs348/en/

[2] M. Li, M. McGuire, K. S. Ho, and G. Hayward, "Array element failure correction for robust ultrasound beamforming and imaging," in Proc. IEEE Int. Ultrason. Symp., Oct. 2010, pp. 29-32.

[3] J. R. Fort, N. S. Neidell, D. J. Morgan, and P. C. Landmeier, "Synthetic aperture ultrasound imaging system," US Patent 5465 722, Nov. 14, 1995.

[4] M. Bae and M. Jeong, "A study of synthetic-aperture imaging with virtual source elements in b-mode ultrasound imaging systems," IEEE Trans. Ultrason., Ferroelect., Freq. Control, vol. 47, no. 6, pp. 1510-1519, Nov. 2000.

[5] J. A. Jensen, S. I. Nikolov, K. L. Gammelmark, and M. H. Pedersen, "Synthetic aperture ultrasound imaging," Ultrasonics, vol. 44, pp. e5-e15, 2006.

[6] S. Korukonda and M. M. Doyley, "Estimating axial and lateral strain using a synthetic aperture elastographic imaging system," Ultrasound Med. Biol., vol. 37, no. 11, pp. 1893-1908, 2011.

[7] N. Bottenus et al., "Feasibility of swept synthetic aperture ultrasound imaging," IEEE Trans. Med. Imag., vol. 35, no. 7, pp. 1676-1685, Jul. 2016.

[8] C. E. Burckhardt, P. Grandchamp, and H. Hoffmann, "An experimental 2 mhz synthetic aperture sonar system intended for medical use," IEEE Trans. Sonics Ultrason., vol. SU-21, no. 1, pp. 1-6, Jan. 1974.

[9] J. Kortbek, J. A. Jensen, and K. L. Gammelmark, "P2b-1 synthetic aperture focusing applied to imaging using a rotating single element transducer," in Proc. IEEE Ultrason. Symp., 2007, pp. 1504-1507.

[10] J. Opretzka, M. Vogt, and H. Ermert, "A synthetic aperture focusing technique with optimized beamforming for high-frequency ultrasound," in Proc. IEEE Int. Ultrason. Symp., 2010, pp. 2303-2306.

[11] J. Opretzka, M. Vogt, S. Maschauer, O. Prante, and H. Ermert, "High frequency ultrasonic imaging based on a combination of synthetic aperture focusing and limited angle compounding: Small animals imaging results," in Proc. Eur. Conf. Synthetic Aperture Radar, 2012, pp. 38-41. 
[12] H. Andresen, S. I. Nikolov, and J. A. Jensen, "Synthetic aperture focusing for a single-element transducer undergoing helical motion," IEEE Trans. Ultrason., Ferroelect., Freq. Control, vol. 58, no. 5, pp. 935-943, May 2011.

[13] J. A. Neasham and D. J. Graham, "Ultrasound imaging apparatus," US Patent 9161 742, Oct. 20, 2015.

[14] C. F. Schueler, H. Lee, and G. Wade, "Fundamentals of digital ultrasonic processing," IEEE Trans. Sonics Ultrason., vol. 31, no. 4, pp. 195-217, Jul. 1984.

[15] K. J. Smith, D. J. Graham, and J. A. Neasham, "Design and optimization of a voice coil motor with a rotary actuator for an ultrasound scanner," IEEE Trans. Ind. Electron., vol. 62, no. 11, pp. 7073-7078, Nov. 2015.

[16] P. Bloomfield, Fourier Analysis of Time Series: An Introduction. New York, NY, USA: Wiley, 2004.

[17] R. N. Czerwinski, D. L. Jones, and W. D. O'Brien, "Detection of lines and boundaries in speckle images-application to medical ultrasound," IEEE Trans. Med. Imag., vol. 18, no. 2, pp. 126-136, Feb. 1999.

[18] J. A. Jensen and N. B. Svendsen, "Calculation of pressure fields from arbitrarily shaped, apodized, and excited ultrasound transducers," IEEE Trans. Ultrason., Ferroelect., Freq. Control, vol. 39, no. 2, pp. 262-267, Mar. 1992.

[19] J. A. Jensen, "Field: A program for simulating ultrasound systems," in Proc. Nordicbaltic Conf. Biomed. Imaging, 1996, pp. 351-353.

[20] J. M. Thijssen, G. Weijers, and C. L. de Korte, "Objective performance testing and quality assurance of medical ultrasound equipment," Ultrasound Med. Biol., vol. 33, no. 3, pp. 460-471, Mar. 2007.

[21] F. P. Hadlock, R. B. Harrist, R. S. Sharman, R. L. Deter, and S. K. Park, "Estimation of fetal weight with the use of head, body, and femur measurementsa prospective study," Amer. J. Obstet. Gynecol., vol. 151, no. 3, pp. 333-337, 1985.

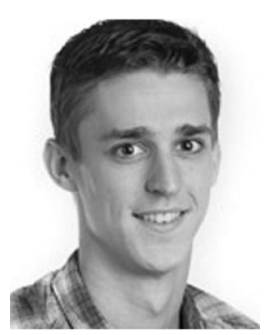

Thomas L. A. van den Heuvel received the B.Sc. and M.Sc. degrees in biomedical engineering from Eindhoven University of Technology, Eindhoven, the Netherlands, in 2012 and 2014, respectively. $\mathrm{He}$ is currently working toward the Ph.D. degree at the Medical Ultrasound Imaging Centre and the Diagnostic Image Analysis Group in Nijmegen, the Netherlands. His Ph.D. project focusses on the improvement of antenatal care diagnostics for the poor by development of a low-cost ultrasound device and computer aided detection software to detect maternal

mortality risk factors.

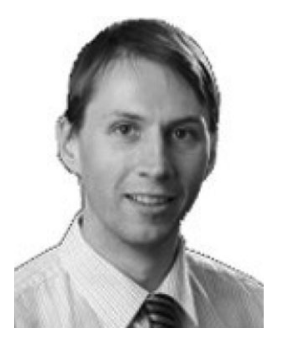

Kristopher J. Smith received the B.Sc.(Hons.) degree in communications and electronic engineering and the M.Sc. degree in microelectronic engineering and the Eng.D. degree in engineering both from Newcastle University, Newcastle upon Tyne, U.K., with a sponsorship from Dyson. His Eng.D. research focused on power supply quality in brushless drives. Prior to attending Northumbria University, he completed an apprenticeship with British Telecom. After completing the Doctorate degree, he moved into a Teaching Fellowship at Newcastle University. His research interests are mainly in permanent-magnet machine design.

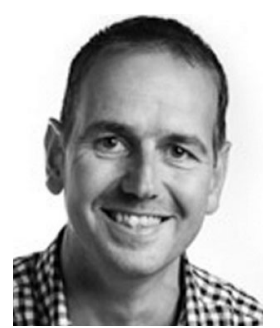

Chris L. de Korte (M'01-SM'15) born in 1969 $\mathrm{He}$ received the M.Sc. degree in medical electrical engineering from Eindhoven University of Technology, Eindhoven, the Netherlands, in 1993. The practical work was performed at the Biophysics Laboratory, Institute of Ophthalmology, University Hospital Nijmegen, the Netherlands, from 1992 to 1993. He studied the acoustical properties of eye tissues. In 1999, he received the Ph.D. degree in medical sciences from the Thoraxcenter, Erasmus University Rotterdam, Rotterdam, the Netherlands, on the subject intravascular ultrasound elastography. From 1999 to 2002, he was Principal Investigator of the project to translate intravascular palpography to clinical applications. In 2002, he joined the Clinical Physics Laboratory, Radboud University Nijmegen Medical Center, Nijmegen, the Netherlands, as an Assistant Professor. In 2004, he became the Head of the laboratory, and from 2006 he has been an Associate Professor of medical ultrasound techniques. He has (co)authored more than 80 peer-reviewed articles in international journals, is (co)inventor of three patents. His main research interests are functional imaging using ultrasound, acoustical and mechanical tissue characterization, and ultrasound image segmentation for cardiac, vascular, muscular, and other applications. He has received the EUROSON Young Investigator Award, 1998, of the European Federation of Societies for Ultrasound in Medicine and Biology. He organized the International Tissue Elasticity Conference, in 2009.

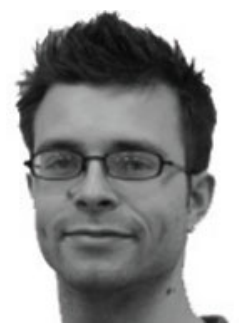

David J. Graham received the B.Eng. degree in electronic engineering and the Ph.D. degree in electronic communications from Newcastle University, Newcastle upon Tyne, U.K., in 2007 and 2011, respectively. His Ph.D. study focused on throughmetal communications and power delivery. Since graduating, he has worked as a Research Associate in the Communications, Sensors, Signal and Information Processing Research Group ( $\left.\mathrm{ComS}^{2} \mathrm{IP}\right)$, Newcastle University. His research interests include acoustic communication, digital signal processing, wireless sensor networks, and acoustic imaging.

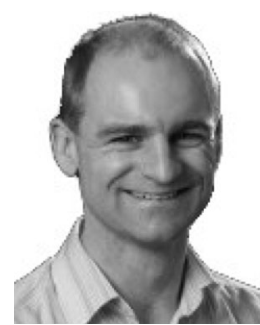

Jeffrey A. Neasham received the B.Eng. degree in electronic engineering from Newcastle University, Newcastle upon Tyne, U.K., in 1994. He then worked at Newcastle University until 2007 as a Research Associate on research and commercial product development in underwater acoustic communication, sonar imaging, and wireless sensor networks, before taking up an academic post. He is currently a Senior Lecturer of communications and signal processing in the School of Electrical and Electronic Engineering, Newcastle University. His research interests include acoustic/ultrasonic signal processing and device design, wireless communication, and biomedical instrumentation. 